\title{
Strengthening HIV services for pregnant women: an opportunity to reduce maternal mortality rates in Southern Africa/sub-Saharan Africa
}

\author{
J Moodley, ${ }^{\text {a }}$ RC Pattinson, ${ }^{\text {b }}$ C Baxter, ${ }^{c}$ S Sibeko, ${ }^{c}$ Q Abdool Karim ${ }^{c}$ \\ a Women's Health and HIV Research Group, Department of Obstetrics and Gynaecology, University of KwaZulu-Natal, Congella, South Africa \\ ${ }^{\mathrm{b}}$ Department of Obstetrics and Gynaecology, University of Pretoria, Pretoria, South Africa ${ }^{\mathrm{c}}$ Centre for AIDS Programme of Research in \\ South Africa (CAPRISA), Nelson R Mandela School of Medicine, University of KwaZulu-Natal, Congella, South Africa \\ Correspondence: Prof. J Moodley, Women's Health and HIV Research Group, Nelson R Mandela School of Medicine, University of KwaZulu- \\ Natal, Private Bag 7, Congella, 4013 South Africa. Email jmog@ukzn.ac.za
}

Accepted 13 August 2010. Published Online 24 September 2010.

Reliable data from South Africa emanating from WHO recommendations for the Safe Motherhood programme underscores HIV/AIDS as the most common cause of maternal deaths. The strengthening of HIV services for pregnant women especially in countries with a high burden of HIV infection will reduce HIV-related and un-related maternal mortality rates. High-quality and complete data on maternal deaths is a critical foundation for reliably monitoring temporal trends in maternal deaths, and causes thereof, but needs substantial strengthening in many resource-constrained settings.

HIV/AIDS is an increasing contributor to direct and indirect causes of maternal deaths in sub-Saharan Africa. A review of published data on maternal deaths and its association with HIV shows that reliable data come from the Confidential Enquiries into Maternal Deaths from South Africa, population-based surveys in sentinel populations, and facility-based data. Despite an increase in knowledge of the HIV status of pregnant women and the initiation of antiretroviral treatment, reversals in trends towards increased maternal deaths are not being observed. The strengthening of HIV services provides an opportunity to alter HIV epidemic trajectories and reduce maternal deaths.

Keywords HIV, maternal mortality, sub-Saharan Africa.

Please cite this paper as: Moodley J, Pattinson R, Baxter C, Sibeko S, Abdool Karim Q. Strengthening HIV services for pregnant women: an opportunity to reduce maternal mortality rates in Southern Africa/sub-Saharan Africa. BJOG 2011;118:219-225.

\section{Introduction}

In a recent commentary in the Lancet, ${ }^{1}$ the editor begged an answer to the question 'what is it going to take to stop mothers dying?' We argue, based on rigorously collected data from South Africa, that the strengthening of HIV services for pregnant women will make a substantial contribution, especially in settings where women bear a disproportionate burden of HIV infection. Notably of the health-related Millennium Development Goals, the least progress has been made on reducing maternal deaths, and indeed some resource-constrained settings have seen reversals in the gains made in the early 1990s.

\section{HIV and maternal deaths}

Despite the already high burden of HIV infection in subSaharan Africa, HIV continues to spread unabatedly. ${ }^{2}$ Each year, there are more than 2 million pregnancies in HIVinfected women in the world, with the majority occurring in sub-Saharan Africa where access to HIV services remain limited. ${ }^{3}$ Every year approximately 250000 African women die during pregnancy, delivery, or the puerperium, accounting for $50 \%$ of the global burden of maternal deaths. $^{4}$

There is a close correlation in sub-Saharan Africa between the increasing burden of HIV infection and the simultaneous increase in maternal deaths, ${ }^{5}$ and there is increasing evidence that HIV infection is becoming the major cause of maternal mortality in resource-constrained settings. The lack of complete data on the causes of maternal deaths for most sub-Saharan countries limits our ability to quantify the attributable risk of HIV/AIDS, or project with accuracy the number of maternal deaths that could be averted by strengthening HIV services for pregnant women. 
Moodley et al.

Recently published global estimates for maternal mortality, based on an assessment of levels and trends in maternal mortality for 181 countries, ${ }^{5}$ highlight the impact of HIV on maternal mortality, and suggest that progress in reducing maternal mortality has been slowed by the HIV pandemic.

\section{Evidence of link between HIV and maternal deaths from South Africa}

In South Africa, the Saving Mothers report, ${ }^{6}$ based on data collected between 2005 and 2007, indicates that HIV/AIDS accounted for approximately $43.7 \%$ of facility-based maternal deaths, and for about a ten-fold increased risk of maternal deaths. This is a conservative estimate, as the HIV status for $37.7 \%$ of the cases were unknown. ${ }^{6}$ Thus, indirect causes of maternal deaths because of HIV/AIDS have surpassed direct obstetric causes (hypertensive disorders of pregnancy, 15.7\%; obstetric haemorrhage, $12.4 \%$; and pregnancy-related sepsis, 9\%) of maternal deaths in South Africa. The process of reporting information on confidential enquires into maternal deaths in South Africa is published in the Saving Mothers report published triennially. ${ }^{6}$

Independently undertaken, single facility-based audits carried out in South Africa have also shown higher rates of maternal mortality in HIV-infected women compared with HIV-uninfected women. ${ }^{7,8}$ Data have been published from two studies at a major tertiary hospital in KwaZulu-Natal (the epicentre of the pandemic): in the first, Khan et al. ${ }^{7}$ in 2001 demonstrated a 32-fold increased risk of death among TB-HIV co-infected women, compared with those without HIV infection; and in the second study, Ramagoale et $a .^{8}{ }^{8}$ in 2007 reported that the majority of deaths among HIV-infected women were associated with co-infection with tuberculosis $(22 \%)$, pneumonia $(19.9 \%)$, and meningitis. More recently, a 5-year review of maternal deaths at a tertiary health facility in Johannesburg, South Africa, shows that maternal mortality ratios in HIV-infected women were 776/100 000 (95\% CI 591-1000/100 000 births), and were 6.2-fold higher (95\% CI 3.6-11.4) than in HIV-negative women (124/100 000; 95\% CI 72-199/100 000 births). ${ }^{9}$ These authors reported that although HIV testing rates increased from 43.4 to $72.2 \%$ during this period, it remained suboptimal, and could explain why although $59.2 \%$ of HIV-infected pregnant women with known HIV status had been initiated on antiretroviral treatment, its impact on life expectancy was not yet evident. They therefore suggested that an updated assessment of the effects of HIV on maternal morbidity is needed, and that an accelerated preparation plan and initiation of HIV treatment may considerably reduce the high maternal mortality figures associated with HIV. ${ }^{9}$
The Saving Mothers report (2005-2007) also found that only $60 \%$ of mothers who died had had an HIV test, and of these, $78.9 \%$ tested positive. The criteria used for assigning cause of death as AIDS in the Saving Mothers report, was very conservative. The women had to have had a positive HIV test and a cluster of differentiation 4 (CD4) count of $<200$ cells $/ \mu$ l or an AIDS-defining condition to minimise bias with respect to a mistaken diagnosis as a cause of maternal death. It is worth noting that for $27.8 \%$ (255/915) of maternal deaths, where cause of death was labelled as AIDS, mothers also had an obstetric condition that could have been assigned as the cause of death. A total of 58 women with AIDS had an abortion, another 16 women with AIDS had the final cause of death reported as hypovolaemic shock associated with postpartum haemorrhage, and an additional 181 women were recorded as having died of septic shock, which could be classified as puerperal sepsis. ${ }^{6}$ Even if these deaths were reclassified as direct causes of maternal deaths, then $78.9 \%$, or about four out of five, maternal deaths where the HIV status was known would still be attributable to HIV. Based on this data, there is no doubt that HIV infection is the largest threat to maternal mortality in South Africa.

Reports from other parts of sub-Saharan Africa have also shown that the risk of maternal death in HIV-infected women is higher than in uninfected women, by as much as five-fold. ${ }^{10-13}$ A 12-month prospective cohort study of the natural history of HIV infection and transmission from mothers to infants, in Malawi, Zambia and Tanzania, ${ }^{11}$ found that there were no deaths among the $331 \mathrm{HIV}$-uninfected women, whereas in 2292 HIV-infected mothers, $42(1.8 \%)$ died. Ninety percent of the mothers died in the postpartum period, and the authors speculate that underlying health status, such as anaemia and malaria, could have influenced this outcome. ${ }^{11}$ In sub-Saharan Africa, anaemia is the leading cause of morbidity, and is mainly caused by malaria, intestinal parasites and HIV. ${ }^{11,12} \mathrm{~A}$ prospective mortuary investigation of all deaths in women aged 1544 years in Pointe Noire, Congo, for a period of 112 consecutive days, indicated that the maternal mortality rate (MMR) was 32 times higher (95\% CI 25-39) among HIVinfected than HIV-uninfected women. ${ }^{14}$ In Uganda and Zimbabwe, a comparison of morbidity and mortality patterns in HIV-infected and uninfected women during pregnancy, and in the subsequent 2 years, showed that HIV-infected women were more likely to be admitted to hospital or die during the 2 years after delivery than the HIV-uninfected pregnant women. ${ }^{15}$ Data from a community randomised trial conducted in the Rakai district of Uganda reported that maternal mortality ratios in HIVinfected mothers was 1686.8/100 000 live births compared with 309.8/100 000 live births in HIV-uninfected women (RR 5.44; 95\% CI 1.98-14.93)..$^{12}$ 
In contrast, in well-resourced countries, because of the relatively small HIV epidemic in women, data on the contribution of HIV to maternal mortality is limited. A study from the USA reported that HIV-infected women undergoing caesarean section were at a significantly greater risk of mortality than uninfected women, and were twice as likely to have one or more postpartum morbidities compared with HIV-uninfected pregnant women (16.1 versus $8.5 \%$, $P<0.001){ }^{16}$

\section{HIV-related maternal deaths: an underestimate?}

A key reason that the data from the Saving Mothers report is an underestimate of true maternal deaths, including those associated with HIV, is because it is based on facilitybased reports, and not all mothers utilise healthcare facilities for antenatal, intrapartum, or postpartum care. ${ }^{6}$

Population level data on maternal deaths are available from two rural sites in South Africa. Vital statistics have been gathered by the Africa Centre from the Hlabisa subdistrict in northern KwaZulu-Natal province, South Africa, in 6-monthly intervals from 2000, and includes about 86000 people in 11000 households. ${ }^{17}$ A follow-up visit is conducted in all households where a death has occurred, and a verbal autopsy is completed to ascertain the cause of death using The International Statistical Classification of Diseases and Related Health Problems 10th Revision (ICD10), as well as circumstances and geographical location of death. The temporal trend analysis of this data (pers. comm., W Graham) from 2000-2007 indicate that of the 11274 deaths that occurred during this period, 151 were maternal deaths (defined as a death up to 90 days following delivery). About two-thirds (62.8\%) of the maternal deaths were in hospital, $31.7 \%$ were at home, and $6 \%$ were unknown/missing data, and $59 \%$ of the deaths occurring at home were to the result of AIDS or tuberculosis. These data are related to deaths up to 90 days following delivery. ${ }^{17}$ Similar population-based surveys from the Agincourt site demonstrated 26 maternal deaths over the period 2000-2007. ${ }^{18}$ Six of these maternal deaths occurred at home $(23.1 \%)$. Based on these two population-based surveys, the number of home deaths in South Africa ranges from 23.1 to $37.7 \%$.

Population-based estimates remain rare globally. Data from other resource-constrained settings demonstrate that maternal deaths that occur at home range from 14 to $68 \%{ }^{19}$

Not surprisingly, the estimates of maternal deaths of $147 / 100000$ live births reported in the Saving Mothers report are conservative.

Using data from the 2001 census of South Africa on external causes and maternal mortality led to estimates of $542 / 100000$ live births. ${ }^{20}$ The authors of the South African census 2001 data state that this level of maternal deaths is much higher than the estimates pre-dating the HIV/AIDS era. The main reasons given for these excessive levels were HIV/AIDS. ${ }^{20}$

\section{Does pregnancy advance HIV disease?}

One question raised by HIV and maternal mortality is the nature of the interaction of HIV with pregnancy. Does the dual immune-suppression from pregnancy and the advancing HIV disease work antagonistically, and lead to a more rapid HIV disease progression? Although the initial views were that increased morbidity and mortality was associated with clinical progression, as adjudged by CD4 counts and viral loads, recent evidence suggests that pregnancy appears to have no discernable effect in increasing the progression of HIV disease in asymptomatic HIV-infected women, ${ }^{21,22}$ but that symptomatic HIV-infected women are at a greater risk of dying from infectious diseases than uninfected pregnant women. The most commonly occurring opportunistic infections in HIV-infected pregnant women in resourceconstrained settings include common treatable infections such as bronchopneumonia, Pneumocystis carinii pneumonia, meningitis, tuberculosis, and malaria. ${ }^{6,23}$ More aggressive efforts to prevent, diagnose, and treat these conditions prior to and during pregnancy will reduce morbidity and mortality in HIV-infected pregnant women.

\section{Strategies for reducing maternal deaths}

Based on the data, enhancing our knowledge of HIV status in pregnant women and their sexual partners, more aggressive diagnosis and treatment of common co-morbidities, and focussing more critically on the postpartum period are the obvious areas that need strengthening. Not surprisingly, the solutions for reducing HIV-related maternal deaths are similar to that for reducing overall maternal mortality, namely establishing political will and working within a human-rights framework that includes maternal rights, strengthening health systems, effective policies, and encouraging community participation and social mobilisation that underscores the importance of saving mothers' lives. $^{24}$ Fortney and Leong ${ }^{24}$ describe a systematic threestep approach to reducing maternal mortality, namely: (1) prevent unwanted pregnancies; (2) prevent complications from occurring during pregnancy, delivery, and the postpartum period; and (3) prevent deaths when complications occur. This approach resonates with the WHO fourcomponent universal strategic plan to reduce transmission of HIV to infants, namely: (1) primary prevention of HIV among women of child-bearing age; (2) preventing unintended pregnancy in HIV-infected women; (3) preventing 
mother-to-child transmission (PMTCT) of HIV during pregnancy; and (4) providing treatment, care, and support for infected women and their families.

\section{Preventing pregnancy}

Each year approximately 80 million women, regardless of HIV status, have unwanted pregnancies. ${ }^{25}$ Strengthening efforts to prevent these unwanted pregnancies, particularly in HIV-infected women, would be the single most effective means of preventing maternal deaths. ${ }^{24}$ This can be achieved through increasing awareness, understanding obstacles to contraceptive use, and making acceptable family planning methods readily accessible and affordable. ${ }^{26-28}$ Aggressive campaigns to promote family planning in continents with high birth rates like Asia and Africa has the potential to avert $150000(32 \%)$ of all maternal deaths globally. ${ }^{26}$ In 2002, despite only $13 \%$ of married women aged 15 49 years in sub-Saharan Africa using modern contraceptive methods, an estimated 22\% (or 173 000) of HIV-positive births were prevented. ${ }^{26}$ The cost effectiveness of family planning programmes to avert HIV-positive births has been assessed using a hypothetical sub-Saharan African population, and demonstrate that for the same costs, the contraceptive strategy could avert $28.6 \%$ more HIV-positive births than the two-dose nevirapine intervention for PMTCT, ${ }^{27}$ and thereby reduce both maternal and infant deaths.

Health systems should maximise access to family planning services and ensure that every opportunity is seized to offer a variety of contraceptive methods. Opportunities such as the time of discharge from maternity units, postnatal clinics, when mothers bring children for immunisations, and the post-abortion and the postpartum periods are also excellent windows of opportunity that should not be missed. It is more effective to discuss contraception at these points than to refer women to another health facility or to ask them to return at a later date. ${ }^{27}$ In cases of HIVinfected women attending dedicated clinics, opportunities must be taken to offer contraception at each visit.

Although unsafe abortion rates have declined by $17 \%$ in the past 10 years (from 35 to 29/1000 women of childbearing age), maternal mortality resulting from abortions remained static at 70000 deaths per year. ${ }^{29,30}$ The Saving Mothers report found that deaths from unsafe abortions have increased since the previous report. Improving access to, and increasing the number of health facilities providing access to legal abortion services would decrease deaths from abortion. ${ }^{6}$ Obviously, access and the availability of services is essential, but so too is the importance of improving the quality of the termination of pregnancy services. Health services must in addition provide contraception at all levels of health care, and provide abortion services at the lowest level of the health service.

\section{Prevention of HIV}

Abstinence, fidelity, the consistent use of condoms, monogamous relationships, awareness of one's HIV status, and more recently, mass male circumcision (MMC) have become the cornerstones of global HIV prevention efforts. Notwithstanding the need for methods that can be initiated by women, knowledge of HIV status is an important gateway to access of prevention and treatment services. Thus, offering HIV testing and counselling to all women whenever an opportunity presents (during the antenatal period, following delivery of the unbooked mother, and at the time of immunisation of the baby), is an important strategy, particularly because voluntary counselling and testing (VCT) has been shown to be effective in reducing risky sexual behaviour, and is costeffective as a preventative intervention. ${ }^{31,32}$ However, large numbers of people in sub-Saharan Africa decline testing because of the fear of stigma and discrimination. These obstacles together with weak laboratory systems, such as the lack of laboratory services for CD4 counts, and the delay in obtaining results, hinder the uptake of VCT in the PMTCT programmes. ${ }^{33}$

Recently, MMC has been promoted as an HIV-preventative strategy based on compelling evidence of protection from three independently conducted randomised controlled trials. $^{34-36}$ This provides a wonderful opportunity to target men for VCT services, and get them more engaged in HIVprevention efforts, including saving mothers and taking greater responsibility as fathers. ${ }^{37}$

\section{Preventing complications}

The prevention of complications plays an important role in obstetric practice and much of the prevention activities have been described previously, and these strategies are instituted during the antenatal, intrapartum, and postpartum periods. ${ }^{21-24}$

Specifically related to HIV-associated morbidity and deaths, is good nutrition, which includes sufficient quantities of iron, iodine, folic acid and vitamin A. Anaemia has been reported to be an important co-factor in HIV-related maternal deaths. ${ }^{11}$ Identifying and treating anaemia is therefore vitally important, not only as a general nutritional measure, but to prevent complications associated with malaria, HIV/AIDS, hepatitis and sickle-cell anaemia. There is also evidence that providing vitamin A to women during pregnancy may, in addition to improving their own health, prevent death, which is most likely to originate in the intrapartum period. ${ }^{38}$

More recently, Kirkwood et al. (2010) did a similar trial to the Nepal study on vitamin A supplementation as a safe motherhood or child survival strategy. Their findings do 
not support the use of low-dose vitamin A supplementation for women in their reproductive phase of life to reduce maternal mortality. ${ }^{39}$

The implementation of obstetric practices such as keeping the amniotic membranes intact during labour, limiting the number of vaginal examinations, performing episiotomies only if necessary, and preventing prolonged labour, may reduce postpartum infections, and therefore postpartum morbidity and death. ${ }^{21,22,40}$ There is some evidence that the use of prophylactic antibiotics in HIV-infected labouring women may reduce postpartum infectious morbidity, particularly in those who had episiotomies. ${ }^{40}$ In a randomised trial conducted in Durban, South Africa, HIVpositive pregnant women in whom vaginal delivery was anticipated, were allocated to receive Cefoxitin or placebo in labour: those that received antibiotics had a 53\% reduction in postpartum endometritis. ${ }^{40}$ However, in a recent placebo-controlled trial, the administration of perinatal and prenatal antibiotics during pregnancy in 1558 women had no effect on maternal mortality and morbidity among HIV-infected pregnant Zambian, Malawian, and Tanzanian women. ${ }^{41}$ Further studies on the use of prophylactic antibiotics in HIV-infected women to reduce maternal mortality are required.

The Saving Mothers report indicates that bacterial pneumonias, bacterial sepsis, atypical pneumonia, cryptococcal meningitis, and tuberculosis are common co-morbid conditions associated with HIV/AIDS, and may be the primary causes of maternal death. ${ }^{6}$ HIV and tuberculosis co-infection has emerged as a substantial contributor to maternal deaths in sub-Saharan Africa. ${ }^{42}$ Tuberculosis is rampant in sub-Saharan Africa, and maternal mortality studies show that tuberculosis was the third leading infectious cause of maternal death in Durban $(14.9 \%)^{7}$ and in Lusaka $(25 \%),{ }^{43}$ where 33 of 36 women were infected with HIV. These observations strongly indicate that in Africa, where antenatal care may be the first encounter a woman has with the health system, effective interventions to screen and treat for these conditions must be prioritised and instituted into routine antenatal packages at all levels of health care. In South Africa, although screening for tuberculosis during pregnancy is recommended, it does not seem to be undertaken routinely. ${ }^{9}$ This may contribute to a delay in diagnosis, and considerable morbidity and even mortality. Screening for malaria and prophylactic anti-malarial therapy in the relevant countries is also of value. ${ }^{42}$ Furthermore, cotrimoxazole prophylaxis has been shown to decrease the number of episodes of pneumonia. Similarly, isoniazid (INH) prophylaxis may be of value. ${ }^{42}$

Postnatal care is poor in African countries, and puerperal sepsis is a major cause of maternal mortality. Following delivery, women should remain in hospital for at least 24 hours or more, and the need for postnatal care and follow-up is essential. Women should be informed of the warning signs of infection, and to return prior to the routine 6-7-day postnatal follow-up visit if necessary. Clinical experience suggests that HIV-infected women may be clinically well at the time of discharge, but are re-admitted with florid sepsis 7-10 days following delivery. ${ }^{6,39}$ Thus strengthening postnatal care in sub-Saharan countries is of paramount importance.

\section{Human resources}

Most, if not all countries, have chronic shortages of human resources for health. In sub-Saharan countries, the HIV epidemic has also had a significant negative impact on healthcare professionals in countries with the highest rates of infection. In Botswana, $17 \%$ of the health workforce died from diseases related to AIDS between 1999 and $2005 .^{44}$ In South Africa, the prevalence of HIV among health professionals approximates that of the general population, leading to higher rates of illness and absenteeism among the very people needed to provide health services. ${ }^{45}$ Combined with a 'brain drain', Samb et al. ${ }^{46}$ state that in a vicious circle, the epidemic fuels the crisis in the health workforce at the same time the shortage of health workers presents a major barrier to preventing and treating the disease'.

Achieving a goal of universal access to comprehensive HIV prevention programmes, treatment, and care and support, however, cannot be achieved without strengthening healthcare systems and the significant expansion of health workforces. One way to expand the health workforce is by 'task shifting', the process of delegation, in which tasks are moved where appropriate from more to less specialised health workers. ${ }^{46}$ This allows for the more efficient use of the available overall workforce. Although the term 'task shifting' is new, the concept of such practices has existed for some time outside HIV-related services. For example, in South Africa, specially trained midwives manage maternity units at a primary health care clinic level. In addition, PMTCT trained nurses and antiretroviral treatment (ART) nurses are the unofficial gatekeepers of HIV knowledge and skills at the primary health care level. ${ }^{47}$ These categories of trained health workers direct HIV testing and counselling services, prepare pregnant women for antiretroviral therapy initiation, and partner with midwives to provide PMTCT services during the perinatal period. ${ }^{47}$ Such practices have been shown to be feasible and acceptable, and are associated with favourable outcomes. ${ }^{48,49}$

\section{Conclusion}

Maternal mortality rates in poorly resourced countries are unacceptably high. In countries with high rates of HIV infection, maternal mortality ratios have increased further, and 
Moodley et al.

HIV/AIDS is now the most common non-obstetric infectious cause of maternal deaths. In sub-Saharan Africa, HIV/AIDS has thus become a human developmental issue, because it diminishes the chances of alleviating poverty, achieving universal primary education, promoting gender equality, and reducing child and maternal mortality. The challenges require a renewed global effort because HIV continues to spread in under-resourced settings, and, globally, women are bearing an increasing burden of HIV infection. Recent reports from the USA highlight the emerging HIV epidemic in minority women at rates comparable with several African countries, ${ }^{50,51}$ and underscore the importance of more global attention and effort being directed at the attributable risk to maternal deaths through HIV.

\section{Disclosure of interests}

The authors have no conflicts of interest.

\section{Contribution to authorship}

All the authors contributed to this article, and all have given permission for publication.

\section{Details of ethics approval}

Not applicable.

\section{Funding}

Support from the World Health Organization (grant no. $2009 / 35299 / 0$ ) for the literature review is gratefully acknowledged.

\section{References}

1 Horton R. What will it take to stop maternal deaths? Lancet 2009;374:1400-2.

2 UNAIDS, WHO. AIDS Epidemic Update. Geneva: Joint United Nations Programme on HIVIAIDS and World Health Organisation, 2009.

3 Berer M. HIV/AIDS, pregnancy and maternal mortality and morbidity: implications for care. In: Berer $M$, Sundari Ravindran TK, editors. Safe Motherhood Initiatives: Critical Issues. London: Reproductive Health Matters, 2000. pp. 194-210.

4 Hill K, Thomas K, AbouZahr C, Walker N, Say L, Inoue M, et al. Estimates of maternal mortality worldwide between 1990 and 2005: an assessment of available data. Lancet 2007;370:1311-9.

5 Hogan MC, Foreman KJ, Naghavi M, Ahn SY, Wang M, Makela SM, et al. Maternal mortality for 181 countries, 1980-2008: a systematic analysis of progress towards Millennium Development Goal 5. Lancet 2010;375:1609-23.

6 National Committee for Confidential Enquiries into Maternal Deaths. Saving Mothers 2005-2007: Fourth Report on Confidential Enquiries into Maternal Deaths in South Africa. Pretoria: National Department of Health, 2009.

7 Khan M, Pillay T, Moodley JM, Connolly CA. Maternal mortality associated with tuberculosis-HIV-1 co-infection in Durban, South Africa. AIDS 2001;15:1857-63.

8 Ramogale MR, Moodley J, Sebiloane MH. HIV-associated maternal mortality - primary causes of death at King Edward VIII Hospital, Durban. S Afr Med J 2007;97:363-6.
9 Black V, Brooke S, Chersich MF. Effect of human immunodeficiency virus treatment on maternal mortality at a tertiary center in South Africa: a 5-year audit. Obstet Gynecol 2009;114:292-9.

10 Bicego G, Boerma JT, Ronsmans C. The effect of AIDS on maternal mortality in Malawi and Zimbabwe. AIDS 2002;16:1078-81.

11 Chilongozi D, Wang L, Brown L, Taha T, Valentine M, Emel L, et al. Morbidity and mortality among a cohort of human immunodeficiency virus type 1-infected and uninfected pregnant women and their infants from Malawi, Zambia, and Tanzania. Pediatr Infect Dis J 2008;27:808-14.

12 Sewankambo NK, Gray RH, Ahmad S, Serwadda D, WabwireMangen F, Nalugoda F, et al. Mortality associated with HIV infection in rural Rakai District, Uganda. AIDS 2000;14:2391-400.

13 van Dillen J, Meguid T, van Roosmalen J. Maternal mortality audit in a hospital in Northern Namibia: the impact of HIV/AIDS. Acta Obstet Gynecol Scand 2006;85:499-500.

14 Le Coeur S, Khlat M, Halembokaka G, Augereau-Vacher C, Batala-M'Pondo G, Baty G, et al. HIV and the magnitude of pregnancy-related mortality in Pointe Noire, Congo. AIDS 2005; 19:69-75.

15 Mbizvo MT, Mmiro FA, Kasule J, Bagenda D, Mahomed K, Nathoo K, et al. Morbidity and mortality patterns in HIV-1 seropositive/seronegative women in Kampala and Harare during pregnancy and in the subsequent two years. Cent Afr J Med 2005;51:91-7.

16 Louis J, Landon MB, Gersnoviez RJ, Leveno KJ, Spong CY, Rouse DJ, et al. Perioperative morbidity and mortality among human immunodeficiency virus infected women undergoing cesarean delivery. Obstet Gynecol 2007;110:385-90.

17 Graham WJ, Newell M, Luabeya KK. Maternal deaths at home. The hidden burden. Int J Gynecol Obstet 2009;107(Suppl 2):S194.

18 Weiner R, Tollman S, Kahn K, Penn-Kekana L. Health and demographic surveillance sites contribute population based data on maternal deaths in rural areas. S Afr Med J 2007;97:944-5.

19 Ronsmans C, Graham WJ. Maternal mortality: who, when, where, and why. Lancet 2006;368:1189-200.

20 Garenne M, McCaa R, Nacro K. Maternal mortality in South Africa in 2001: from demographic census to epidemiological investigation. Popul Health Metr 2008;6:Doi: 10.1186/478-7954-6-4.

21 Mclntyre J. Mothers infected with HIV. Br Med Bull 2003;67: 127-35.

22 Guidozzi F, Black V. The obstetric face and challenge of HIV/AIDS Clin Obstet Gynecol 2009;52:270-84

23 Walson JL, Brown ER, Otieno PA, Mbori-Ngacha DA, Wariua G, Obimbo EM, et al. Morbidity among HIV-1-infected mothers in Kenya: prevalence and correlates of illness during 2-year postpartum follow-up. J Acquir Immune Defic Syndr 2007;46:208-15.

24 Fortney JA, Leong M. Saving mothers lives: programs that work. Clin Obstet Gynecol 2009;52:224-36.

25 The Alan Guttmacher Institute. Sharing Responsibility: Women, Society and Abortion Worldwide. New York and Washington: The Alan Guttmacher Institute, 1999.

26 Cleland J, Bernstein S, Ezeh A, Faundes A, Glasier A, Innis J. Family planning: the unfinished agenda. Lancet 2006;368:1810-27.

27 Reynolds HW, Steiner MJ, Cates W Jr. Contraception's proven potential to fight HIV. Sex Transm Infect 2005;81:184-5.

28 World Health Organisation. Unsafe Abortion - Global and Regional Estimates of the Incidence of Unsafe Abortion and Associated Mortality in 2003. Geneva, Switzerland: World Health Organisation, 2007

29 Singh S, Wulf R, Hussain R, Bankole A, Sedgh G. Abortion Worldwide: A Decade of Uneven Progress. New York: The Guttmacher Institute, 2009 
30 Healy J, Otsea K, Benson J. Counting abortions so abortion counts: indications for monitoring the availability and use of abortion care services. Int J Gynecol Obstet 2006;95:209-20.

31 Sweat M, Gregorich S, Sangiwa G, Furlonge C, Balmer D, Kamenga C, et al. Cost-effectiveness of voluntary HIV-1 counselling and testing in reducing sexual transmission of HIV-1 in Kenya and Tanzania. Lancet 2000;356:113-21.

32 The VCT efficacy study group. Efficacy of voluntary HIV-1 counselling and testing in individuals and couples in Kenya, Tanzania, and Trinidada: a randomised trial. Lancet 2000;356:103-12.

33 Orie EF, Songca PP, Moodley J. An audit of PMTCT services at a regional hospital in South Africa. S Afr Fam Pract 2009;51:492-5.

34 Auvert B, Taljaard D, Lagarde E, Sobngwi-Tambekou J, Sitta R, Puren A. Randomized, controlled intervention trial of male circumcision for reduction of HIV infection risk: the ANRS 1265 Trial. PLOS Med 2005;2:e298.

35 Bailey RC, Moses S, Parker CB, Agot K, Maclean I, Krieger JN, et al Male circumcision for HIV prevention in young men in Kisumu, Kenya: a randomised controlled trial. Lancet 2007;369:643-56.

36 Gray RH, Kigozi G, Serwadda D, Makumbi F, Watya S, Nalugoda F, et al. Male circumcision for HIV prevention in men in Rakai, Uganda: a randomised trial. Lancet 2007;369:657-66.

37 Titus MJ, Moodley J. Snip and prevent! medically performed circumcision - a strategy for reducing the transmission of HIV. S Afr J Obstet Gynaecol 2008;14:66-8.

38 West KP Jr, Katz J, Khatry SK, LeClerq SC, Pradhan EK, Shrestha SR, et al. Double blind, cluster randomised trial of low dose supplementation with vitamin A or beta carotene on mortality related to pregnancy in Nepal. The NNIPS-2 Study Group. BMJ 1999;318:570-5.

39 Kirkwood BR, Hurt L, Amenga-Etego S, Tawiah C, Zandoh C, Danso $S$, et al. Effect of vitamin A supplementation in women of reproductive age on maternal survival in Ghana (ObaapaVitA): a cluster-randomised, placebo-controlled trial. Lancet 2010;375:1640-9.

40 Sebitloane HM, Moodley J, Esterhuizen TM. Prophylactic antibiotics for the prevention of postpartum infectious morbidity in women infected with human immunodeficiency virus: a randomized controlled trial. Am J Obstet Gynecol 2008;198:189.
41 Aboud S, Msamanga G, Read JS, Wang L, Mfalila C, Sharma U, et al. Effect of prenatal and perinatal antibiotics on maternal health in Malawi, Tanzania, and Zambia. Int J Gynaecol Obstet 2009; 107:202-7

42 Grange J, Adhikari M, Ahmed Y, Mwaba P, Dhada K, Hoelscher M, et al. Tuberculosis in association with HIVIAIDS emerges as a major non-obstetric cause of maternal mortality in Sub-Saharan Africa. Int J Gynecol Obstet 2010;108:181-3.

43 Ahmed Y, Mwaba P, Chintu C, Grange JM, Ustianowski A, Zumla A. A study of maternal mortality at the University Teaching Hospital, Lusaka, Zambia: the emergence of tuberculosis as a major nonobstetric cause of maternal death. Int J Tuberc Lung Dis 1999;3: 675-80.

44 HIVIAIDS and Work: Global Estimates, Impact and Response. (Accessed at: www.ilo.org/public/english/protection/trav/aids/activities/ research-policy.htm). Accessed 21 April 2010.

45 Connelly D, Veriava Y, Roberts S, Tsotetsi J, Jordan A, DeSilva E, et al. Prevalence of HIV infection and median CD4 counts among health care workers in South Africa. S Afr Med J 2007;97:115-20.

46 Samb B, Celleleti F, Holloway J, Van Damme W, De Cook KM, Dybul M. Rapid expansion of the health workforce in response to the HIV epidemic. N Engl J Med 2007;357:2510-3.

47 Dohrn J, Nzama B, Murrman M. The impact of the HIV scale up on the role of nurses in South Africa: time for a new approach. J Acquir Immune Defic Syndr 2009;52:527-9.

48 Morris MB, Chapula BT, Chi BH, Mwango A, Chi HF, Mwanza J, et al. Use of task shifting to rapidly scale-up HIV treatment services: experiences from Lusaka, Zambia. BMC Health Serv Res 2009;9:5.

49 Hussein J, Newlands D, D'Ambruoso L, Thaver I, Talukder R, Besana G. Identifying practices and ideas to improve the implementation of maternal mortality reduction programmes: findings from five South Asian countries. BJOG 2010;117:304-13.

50 El-Sadr WM, Mayer KH, Hodder SL. AIDS in America - forgotten but not gone. N Engl J Med 2010;362:967-70.

51 Center for Disease Control and Prevention. Subpopulation Estimates From the HIV Incidence Surveillance System - United States, 2006. MMWR 2008;57:985-9. 